\section{Microfluidic Optical Devices}

Barbaros Cetin $^{1}$, Soheila Zeinali ${ }^{2}$ and

Dongqing $\mathrm{Li}^{3}$

${ }^{1}$ Mechanical Engineering Department, Bilkent University, Ankara, Turkey

${ }^{2}$ Mechanical Engineering Department, Ihsan

Dogramaci Bilkent University, Ankara, Turkey

${ }^{3}$ Department of Mechanical and Mechatronics

Engineering, Faculty of Engineering, University

of Waterloo, Waterloo, ON, Canada

\section{Definition}

Microfluidic optical devices (MOD) are the emerging technology that combines today's microfluidics technology with the optics. However, MOD can be classified as the integration of these two technologies rather than combination of them. This integration provides a new approach for using microfluidics for control and manipulation of samples and optics for sensing. In this entry we propose a comprehensive review of emerging applications for microfluidic optical devices.

\section{Overview}

In many of the biological applications, microfluidics and optics technology have already been used in combination - microfluidics for control and manipulation of the samples and optics for sensing. Microelectromechanical systems (MEMS) and lab-on-a-chip communities try to embed optical devices into their microsystems to improve functionality of their devices. Presenting a comprehensive review of all the research in the field of microfluidic and optics integration is out the scope of this entry, but several numbers of representative examples of this integration are provided. Moreover, this integration results in handing:

- Electric field induction by light exposure [1]

- Integrated detection systems for microfluidic application (which increases the portability of the entire system) [3-5]
- Sensitivity increase for the existing detection system [7, 8]

- Cell manipulation $[9,11,12]$

- Small cell population sorting with high accuracy by tunable optical fibers [12]

- Rapid detection of environmental contaminants [13]

- A platform to study mammalian individual axonal injury [14]

- Variable-focus liquid lens [15]

\section{Basic Methodology}

Latest developments related to the combination of integrated optics and microfluidics have evolved towards device miniaturization with the ultimate goal of integrating many optical components onto a compact microchip, producing photonic integrated circuits with low cost and high degrees of functionality. Several configurations have been explored in the literature regarding to microfluidic optical devices which can be categorized as below:

- Integration of microtoroid whispering gallery mode (WGM) sensor into a microfluidic system for transporting molecules directly towards the most sensitive area of the sensor [5]

- Combination of optical tweezers and microfluidic chip technologies based on dynamic fluid and dynamic light pattern, in which single and multiple laser traps are employed for cell transportation [12]

- Fabrication of a reticulocyte microfluidic cytometer system based on optimized epifluorescence with the advantage in the signal-to-noise ratio [9]

- All-optical and electrode-free approach into microfluidic chips for achieving reconfigurable dielectrophoresis (DEP) particle trapping [1]

- A detective system by integration of fluorescence detector based on a microavalanche photodiode into a PDMS microfluidic device [3, 4]

- Implementing optical fibers to improve the performance of fluorescent spectroscopy detection on a portable chip [6]

- A neuro-optical microfluidic platform to study mammalian individual axonal injury and subsequent regeneration [14] 


\section{Key Research Findings}

Mccio et al. [1] presented all-optical and electrode-free approach for achieving reconfigurable dielectrophoresis (DEP) particle trapping into microfluidic chips. They used direct laser projection through a holographic spatial light modulator (SLM) onto photorefractive crystal substrates as a fabrication method. As a fabrication process, firstly, they considered an all-optical mold-free approach for fabricating the PDMS microfluidic channel. Secondly, they created geometrical flexible DEP traps onto the substrate by the same SLM holographic projection system. For the first time, they presented the possibility of fabricating microfluidic chips onto lithium niobate (LN) crystals by using the photorefractive effect. In addition, they demonstrated that these PDMS structures can be used as microfluidic devices such as making PDMS chambers and channels in which particles can be trapped by DEP effect.

Chabinyc et al. [2] succeeded the integration of fluorescence detector based on a microavalanche photodiode into a PDMS-based microfluidic device. Their detective system was reusable, and the microfluidic device was disposable. Elimination of the index matching problem which can be problematic in some micromachined devices, the elimination of the need for collection optics, and the inexpensiveness are the superiority of the design. They also addressed some further improvements for the performance of the device.

Mazurczyk et al. [3] fabricated an integrated fluorescence detection system with the microfluidic lab-on-a-chip device. Various arrangements were tested for the fluorescence beam detection like free space optics, fiber optics, and fully waveguiding optics. Free space optics was found to have the higher sensitivity, but it needed the bulk microscope-based detection system. Fiber and fully waveguiding optics seemed to be the possible option to overcome the bulk microscope setup, but their sensitivity was not found to be sufficient especially for high sensitivity required applications. Anyhow, they showed the feasibility of their device for electrophoretic separations by performing some preliminary experiments. They proposed usage of the soda lime glass for the fabrication of their device and envisaged to fabricate more sophisticated systems.

Optofluidics is marriage of fluidics with optics for dynamic manipulation of optical properties at the microscale with applications ranging from photonic circuits to fluidically adaptable optics (Optofluidics). Heng et al. [4] developed a novel on-chip microscope system, which is called optofluidic microscope (OFM). The feasibility of OFM was demonstrated. Their images were comparable to that of a conventional microscope. They suggested the possible use of multiple OFM onto a single microfluidic chip either for increasing imaging throughput in case of parallel usage or sequential imaging of the same target in case of serial usage.

Khosla et al. [5] demonstrated a microfluidic whispering gallery mode (WGM) biosensing system for easy delivery and detection of target molecules. WGM resonators are optical sensors with unprecedented sensitivity because of their small mode volume. Their results showed that the integration of microfluidics and WGM sensing result in highly tunable system, with yield for a given concentration and optimizable sensing time by changing the input power and flow features of the microfluidic system. By combining microfluidics and WGM, particles and molecules as tiny as a single BSA protein (roughly $6 \mathrm{~nm}$ in radius) can be detected, and the analysis can be accomplished for femtomolar concentrations.

Camou et al. [6] used 2D-optical, PDMS lenses to improve the performance of fluorescent spectroscopy detection performed on a portable chip using optical fibers. The fibers are directly inserted into channels ending with PDMS optical lenses. Compared to conventionally use flat interface, these optical lenses increased the intensity of fluorescent response close to the fiber which leads to a higher sensitivity of the on-chip detection method for fluorescent spectroscopy. Chen et al. [7] implemented a simple, on-chip arrayedwaveguide excitation and detection scheme based on the scattering. Detected signals were processed, and not only sensitivity enhancement 
was observed but particles moving with different velocities were also detected accurately.

Kruger et al. [8] proposed a miniaturized flow cytometer using latest photonics technology to perform detection, enumeration, and sorting of fluorescent species. They successfully performed the sample injection, single file flow through the detection system, sorting of fluorescent microbeads. They could not achieve the fully autonomous cell sorting, but they gave that for the future direction. They also demonstrated the feasibility of high gain avalanche photodiodes for more sensitive measurements of fluorescent signals compared to conventional detection techniques.

Ju et al. [9] described design and fabrication of microfluidics reticulocyte cytometer, which can be used for clinical diagnostic and physiology laboratory tests. In addition to the advantages of accuracy and efficiency for application research, the cytometer system of this prototype is based on optimized epifluorescence which has a competitive advantage in the signal-to-noise ratio. To validate microfluidics cytometer, $10 \mu \mathrm{m}$ PS (polystyrene) beads and model of artificial induced anemia mice was used for analyzing. They also addressed a further improvement to fulfill system's potential in detecting reticulocyte maturity. The schematic view of their designed microchip is shown in Fig. 1.

Optical tweezers are the devices that use the force of the strongly focused light to trap and move small objects whose dimensions are below tens of micrometers. The exerted force range and the resolution for optical tweezers are very suitable for biological and macromolecular systems. They have been used in many tasks [10]. A detailed review on optical tweezers can be found elsewhere [10]. Besides optical tweezers, Mandal and Erickson [11] illustrated an optical transport of microparticles in a liquid core waveguiding structure over long lengths, which has a great potential for much more precise particle separation and particle transport without flow filed manipulations. The light-particle interaction length in their proposed technique was orders of magnitude larger than the existing systems. Wang et al. [12] succeeded the integration of optical tweezers and microfluidic chip technologies to handle high accuracy small cell population isolation which exhibits the advantages of noninvasive cell contact and minimal contamination in the isolation and manipulation processes of a generic single. The reported cell sorter has three unique characteristics as exhibiting high purity and high recovery rate in handling small sample population sorting, designing on the analysis of dynamic fluid and the dynamic light pattern, and recognizing the multiple features of cells which refers to the unique capability of the sorter. The sample flow and buffer flow are injected into the microchannel from inlets. A CCD camera is used to take images of cells. For detecting target cells, digital image processing technique is used. After detecting and recognizing target cells, a control signal is generated to position the optical trap on the target cell, and the cell is carried from the sample flow
Microfluidic Optical

Devices, Fig. 1 Sketch of the microchannel device [9]

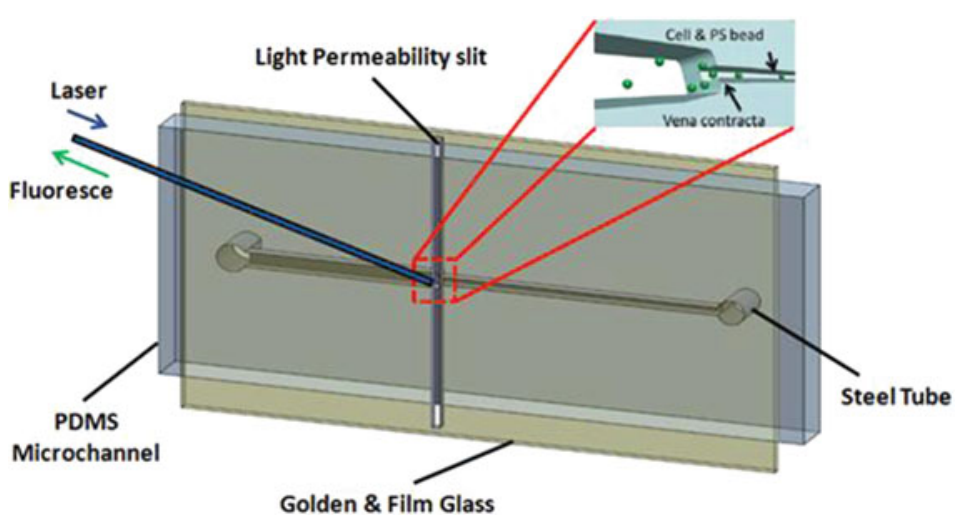




\section{Microfluidic Optical Devices,}

Fig. 2 Schematic of the cell operation principle (Reprinted from [12] with permission from the Royal Society of Chemistry)

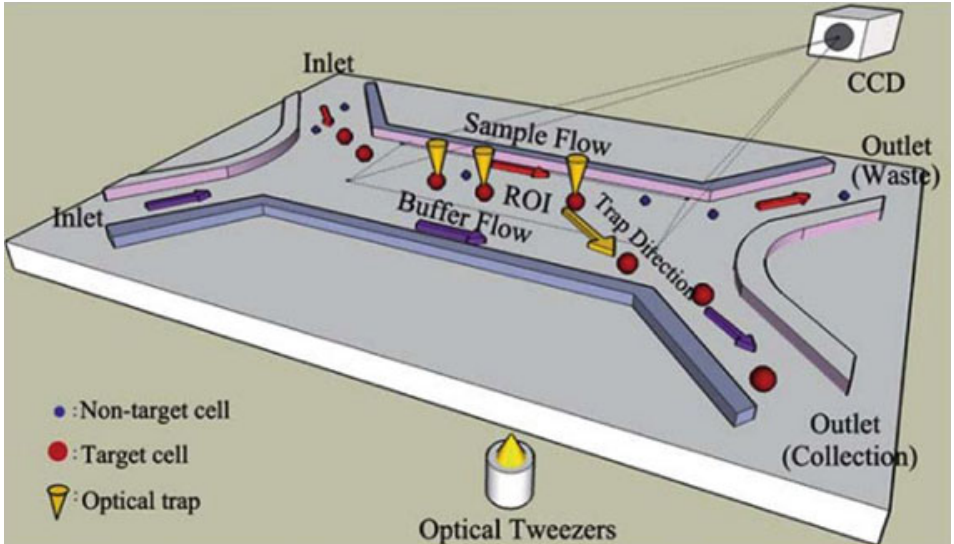

to the buffer flow. When the target cell arrives in the desired position, the optical trap releases the cell, and the cell flows into the outlet along with the buffer flow. The other cells flow into the outlet as waste along with the sample flow. The schematic of the procedure and system setup is shown in Fig. 2 [12].

Lafleur et al. [13] improved conventional methods of environmental analysis by demonstrating the vast potential of gold nanoparticlebased microfluidic sensors for the rapid detection of two important categories of environmental contaminants - heavy metals and pesticides. Using gold nanoparticle-based microfluidic sensors integrated with a simple fluorescence detector allows the detection range of concentrations as low as $0.6 \mu \mathrm{g} / \mathrm{L}$ up to at least $200 \mu \mathrm{g}$; a simple fluorescence detector allows the detection range of concentrations as low as $0.6 \mu \mathrm{g} / \mathrm{L}$ up to at least $200 \mu \mathrm{g} / \mathrm{L}$. These results show that, synergistically, combination of the unique optical properties of gold nanoparticle probes with the inherent qualities of microfluidic platforms offers sensitive sensors for environmental contaminants.

Kim et al. [14] proposed a neuro-optical microfluidic platform to study mammalian individual axonal injury and subsequent regeneration. They succeeded the integration of a compartmentalized neuronal culture microfluidic chip for isolating individual axons, femtosecond laser to have precise and reproducible axotomy, and a mini-incubator to monitor the sequence of neuronal activities and post injury events. In addition to study injury to single axons, the platform can also be used to create unique in vitro neuronal circuit models.

Another interesting application of the microfluidics and the optics is the use of variablefocus liquid lenses, which was developed by Philips Research Eindhoven [15]. The lenses which are composed of two immiscible liquids of different refractive indices can be manipulated by the electrowetting. By electrowetting, the meniscus curvature of the lenses can be changed and so does the effective focal length of the lens.

\section{Future Directions for Research}

Integration of microfluidics and optics merges many advantages associated with these fields and has found many applications in different areas of science and technology. Although, this merging brings many advantages, it also introduces many challenges, and many researchers will continue to solve these challenges and further develop the MODs in the upcoming decades. Microfluidic and microoptical components can be fabricated during the same fabrication step or they can be fabricated separately and postassembled. Despite recent demonstrations, complete integration of both components is still in a highly developmental stage, and exploration of optofluidic functionality is the goal of the most current investigation. 
In addition to the developmental considerations on combination of these two platforms, further investigations on neuro-optical microfluidic platforms are highly recommended because this platform will enable the study and understanding of neuronal response to injury that is currently not possible with conventional cell culture platform and tools.

\section{References}

1. Miccio L, Memmolo P, Grilli S, Ferraro P (2012) All-optical microfluidic chips for reconfigurable dielectrophoretic trapping through SLM light induced patterning. Lab Chip 12:4449-4454

2. Chabinyc ML, Caiu DT, McDonald JC, Stroock AD, Christian JF, Karger AM, Whitesides GM (2001) An integrated fluorescence detection system in poly (dimethylsiloxane) for microfluidic applications. Anal Chem 18:4491-4498

3. Mazurczyk R, Vieillard J, Bouchard A, Hannes B, Krawczyk S (2006) A novel concept of the integrated fluorescence detection system and its application in a lab-on-a-chip microdevice. Sens Actuator B 118:11-19

4. Heng X, Erickson D, Baugh LR, Yaqoob Z, Sternberg PW, Psaltis D, Yang C (2006) Optofluidic microscopy - a method for implementing a high resolution optical microscope on a chip. Lab Chip 6:1274-1276

5. Khosla K, Swaim JD, Knittel J, Bowen WP (2010) Yield enhancement in whispering gallery mode biosensors: microfluidics and optical forces. Journal of Modern Optics 61(5):415-418

6. Camou S, Fujita H, Fujii T (2003) PDMS 2D optical lens integrated with microfluidic channels: principle and characterization. Lab Chip 3:40-45

7. Chen C-H, Tsai F, Lien V, Justis N, Lo Y-H (2007) Scattering-based cytometric detection using integrated arrayed waveguides with microfluidics. IEEE Photon Technol Lett 19(6):441-443

8. Kruger J, Singh K, O’Neill A, Jackson C, Morrison A, O'Brien P (2002) Development of a microfluidic device for fluorescence activated cell sorting. J Micromech Microeng 12:486-494

9. Ju Y, Song J, Geng Z, Zhang H, Wang W, Xie L, Yao W, Li Z (2012) A microfluidics cytometer for mice anemia detection. Lab Chip 12:4355-4362

10. Grier DG (2003) A revolution in optical manipulation. Nature 424:21-27

11. Mandal S, Ericson D (2007) Optofluidic transport in liquid core waveguiding structures. Appl Phys Lett 90(184103): 1-3

12. Wang X, Chen S, Kong M, Wang Z, Costa KD, Li RA, Sun D (2011) Enhanced cell sorting and manipulation with combined optical tweezer and microfluidic chip technologies. Lab Chip 11:3656-3662

13. Lafleur JP, Senkbeil S, Jensen TG, Kutter JP (2012) Gold nanoparticle-based optical microfluidic sensors for analysis of environmental pollutants. Lab Chip 12:4651-4656

14. Kim Y, Karthikeyan K, Chirvi S, Dav DP (2009) Neuro-optical microfluidic platform to study injury and regeneration of single axons. Lab Chip 9:2576-2581

15. Psaltis D, Quake SR, Yang C (2006) Developing optofluidic technology through the fusion of microfluidics and optics. Nature 442:381-386

\section{Microfluidic Platform for Human Diseases}

Nishanth V. Menon, Peng Xue and Yuejun Kang School of Chemical and Biomedical Engineering, Nanyang Technological University, Singapore

\section{Synonyms}

Microfluidic platforms for medical diagnostics; Microfluidics for cancer studies; On-chip disease studies; On-chip drug discovery

\section{Definition}

Microfluidics is an area where the fluid flow and its properties are manipulated in a micron scale for various applications. Some of the microfluidic techniques developed for biological studies have been primarily focused to study, understand, and develop possible diagnostic tools and remedies for human diseases. In the last decade, research in this area has developed rapidly and extensively owing to the possibility of creating and controlling a microenvironment by the enabling microfluidic-based lab-on-a-chip technology, which can mimic the cellular microenvironment in vivo and hence perform individual cell-based studies that are not possible in an actual in vivo model. 